\title{
Identifying target cells for a tick-borne virus that causes fatal hemorrhagic fever
}

\author{
Satoko Yamaoka, Carla Weisend, and Hideki Ebihara
}

Department of Molecular Medicine, Mayo Clinic, Rochester, Minnesota, USA.

\begin{abstract}
Severe fever with thrombocytopenia syndrome (SFTS) is an emerging disease in China, South Korea, and Japan caused by the tick-borne SFTS virus (SFTSV). Severe and fatal SFTS presents as a hemorrhagic fever characterized by high viral load, uncontrolled inflammatory response, dysregulated adaptive immunity, coagulation abnormalities, hemorrhage, and multiorgan failure with up to $33 \%$ case fatality rates (CFRs). Despite its public health significance in Asia, vaccines and specific therapeutics against SFTS are still unavailable. A better understanding of the pathogenesis of SFTS is crucial to improving medical countermeasures against this devastating disease. In this issue of the $J C I$, Suzuki and colleagues analyzed histopathological samples from 22 individuals who succumbed to SFTS, and identified antibody-producing B cell-lineage plasmablasts and macrophages as principal target cells for SFTSV infection in fatal SFTS. Their results suggest that SFTSV-infected post-germinal center B cells, plasmablasts, and macrophages affect systemic immunopathology and dysregulation, which likely leads to fatal outcomes.
\end{abstract}

process at the molecular and cellular level is required, yet relatively little research into these processes has been undertaken.

\section{The main targets of SFTSV infection and replication}

In this issue of the JCI, Suzuki et al. answered the vital question of what types of cells are the main targets of SFTSV infection and replication, which is a crucial clue to understanding the pathogenesis of SFTS (11). The authors clearly demonstrated that antibody-producing B cell-lineage plasmablasts and macrophages are principal targets for SFTSV infection at the terminal stage of SFTS (11). This remarkable finding was achieved by a comprehensive analysis using robust pathology data obtained from autopsy samples of 22 individuals who succumbed to SFTS. First, using immunohistochemistry (IHC) with an anti-SFTSV nucleocapsid protein antibody, the authors demonstrated that $90 \%$ of hematopoietic cells in the secondary lymphoid organs (SLOs), such as spleen and lymph nodes (LNs), are positive for SFTSV antigen, which was consistent with the high viral RNA load detected by RT-PCR in these tissues. Further examination, based on comprehensive IHC for cellular and SFTSV markers in combination with fluorescence in situ hybridization (FISH) for the detection of viral RNA, demonstrated that SFTSV actively replicates in macrophages and mature B cells whose immunophenotype is similar to that of plasmablasts prior to terminal differentiation to plasma cells in LNs (11).

Involvement of multiple organ systems including nonlymphoid organs such as liver, adrenal glands, intestine, lungs, heart, kidneys, and brain is an important pathological feature of fatal SFTS $(6,7)$. Indeed, infectious virus (and viral RNA) has been detected in systemic organs, although the source(s) of infectious virus in these organs has been undefined (6). Notably, the data presented by Suzuki et al. showed that parenchymal cells (i.e., 
endothelial cells and epithelial cells such as hepatocytes in liver) were not infected, but instead, numerous infected B cells at various stages prior to plasmablastlineage differentiation infiltrated into the capillaries in the nonlymphoid organs (11). This is a remarkable and distinct pathological feature of SFTS compared with other VHFs; most VHFs, including Ebola virus disease and Crimean-Congo hemorrhagic fever, involve direct interactions between virus and parenchymal cells (i.e., hepatocytes and endothelial cells) in addition to the cells of the mononuclear phagocytic system (MPS) (monocytes, macrophages, and dendritic cells [DCs]), which are the primary targets for hemorrhagic fever virus (HFV) infection $(12,13)$. A predominant B cell tropism of SFTSV is a clearly distinct pathogenic feature from that of other viruses causing VHFs, and hemorrhagic fever-like syndrome induced by the viral infection in B cells is also rarely seen in humans. In the Suzuki et al. study, the authors showed that not only B cells in the histopathological samples but also PBL-1 cells, which are derived from plasmablastic lymphoma, supported viral multiplication in vitro (11). This result further validates the unique B cell tropism of SFTSV. Collectively, their findings strongly suggest that immune cells, especially plasmablastic B cells and macrophages, are the sole significant effector cells leading the systemic pathology in SFTS.

\section{Inadequate B cell humoral response}

How does the B/plasmablastic cell tropism of SFTSV relate to SFTS pathogenesis? Recently, Song et al. reported that adaptive immune deficiency due to the disruption of B cell-mediated humoral immunity is a hallmark of fatal-SFTS outcome (9). Patients with fatal SFTS failed to mount an immunoglobulin gamma (IgG) antibody response, which is essential to neutralize and clear the virus. To gain further insights into SFTSV-mediated humoral immunity disruption, Suzuki and colleagues next investigated the expression of immunoglobulin (i.e., IgM and $\operatorname{IgG}$ ) in SFTSV-infected $B$ cells in the LNs. The authors used IHC and quantitative RT-PCR to detect mRNA encoding gamma (IGHG) and $\mathrm{mu}(I G H M)$ heavy chains. The results suggested that a majority of the infected B cells were classswitched post-germinal center B cells, and those IgG-positive class-switched B cells also infiltrated systemic organs (11). These findings partially contradict the Song et al. finding, in that a large fraction of plasmablasts in PBMCs from patients with fatal SFTS did not express IgM and IgG, suggesting that activated and differentiated B cells (i.e., plasmablasts and plasma cells) failed to IgG class switch, potentially explaining the inadequate $\mathrm{B}$ cell humoral response in fatalities (9).

Notably, as Suzuki et al. discussed in the present paper, their previous study failed to detect SFTSV-infected cells in the PBMCs from SFTS patients with peripheral blood plasmacytosis (14). Thus, these two studies suggest that there is a large population of noninfected, yet nonfunctional plasmablasts in the peripheral blood of patients with SFTS $(9,14)$. Disease-associated plasmablasts can produce proinflammatory mediators such as interleukin 6, which is a highly expressed proinflammatory cytokine in patients with fatal SFTS, and contribute to inducing systemic inflammation $(7,8,15)$. Notably, massive plasmacytosis in the peripheral blood of patients has also been observed in the severe VHFs such as Ebola virus disease and hantavirus disease $(16,17)$. Therefore, the noninfected, nonfunctional plasmablast population causing plasmacytosis in patients with SFTS $(9,14,18,19)$ may play immunopathological roles in fatal SFTS, or result from immunological dysregulation (i.e., SIRS) induced by SFTSV infection. On the other hand, SFTSV efficiently infects and replicates in the post-germinal center B cells, which are plasmablasts prior to terminal differentiation to plasma cells (11). Another important question, therefore, is whether IgG-positive class-switched B cells infected with SFTSV are still functional to produce IgG antibodies and contribute to viral clearance and disease recovery. Since neutralizing IgGs were undetected in the patients with fatal SFTS (9), IgG-positive class-switched B cells infected with SFTSV are most likely defective and unable to produce functional and/or neutralizing antibodies against SFTSV. It will be important to determine biological and immunological phenotypic differences between two characteristic plasmablast populations, peripheral circulating noninfected plasmablasts and tissue-associated plasmablasts infected with SFTSV.

Interestingly, the plasmablasts in the PBMCs isolated from healthy donors showed surprisingly low susceptibility to SFTSV replication, indicating that normal plasmablasts are missing a cellular factor(s) that makes them vulnerable to SFTSV infection. In accordance with this finding, plasmablastic lymphoma PBL-1 cells exhibited lower susceptibility to SFTSV infection than human macrophage THP-1 cells. The authors, thus, hypothesized that plasmablasts/B cells lack the surface receptor(s) necessary to enhance SFTSV entry into plasmablasts/B cells. Indeed, they found that PBL-1 and other B cell lines lacked the surface expression of DC-SIGN, which is C-type lectin identified as an SFTSV cellular receptor (20). Further, overexpression of DC-SIGN in PBL-1 cells markedly increased SFTSV infection. However, the complicated result that DC-SIGN was not detected in the infected cells in the LNs obtained from deceased individuals suggests that DC-SIGN is dispensable for SFTSV infection at terminal stage of the disease. Germinal center B cell differentiation to plasmablasts during SFTSV infection may lead to the expression of other unknown receptor(s)/factors that support efficient SFTSV entry and/or replication in B cells in vivo.

\section{Primary target cells for the early-stage SFTSV infection}

Collectively, there may be other cell type(s) supporting primary infection of SFTSV prior to the robust viral replication in plasmablasts/B cells. Macrophages and DCs are candidates for the primary target cells for the early stage of SFTSV infection. Indeed, the authors detected SFTSV infection in macrophages in the LNs, although to a lesser extent compared with plasmablasts/B cells. Numerous studies have indicated that HFV infects and activates monocytes/ macrophages, which are the primary target cells for HFV, leading to the expression of proinflammatory cytokines/chemokines (21). Moreover, it has been strongly postulated that HFVs activate macrophages that in part trigger SIRS in severe/fatal VHFs including SFTS $(7,8,15,21)$, and play a critical role in inducing endothelial dysfunction, vascular leakage, coagulation abnormalities, hemophagocytic lympho- 
histiocytosis (HLH), and multiorgan failure (21). In fact, several studies, including that by Suzuki et al., have shown that autopsy pathology of fatal SFTS includes HLH in the LNs, bone marrow, and liver, which is defined as the engulfing of erythrocytes, leukocytes, and platelets by abnormally activated macrophages that are known to cause severe thrombocytopenia, lymphocytopenia, and multiorgan dysfunction/damage $(22,23)$. Importantly, the patients with fatal SFTS displayed prominent hemophagocytosis by uninfected macrophages, strongly suggesting that the virus causes bystander activation due to a systemic, massive inflammatory response. Notably, both groups of hemophagocytic, noninfected macrophages and infected macrophages expressed CD163 (macrophage scavenger receptor) on the cell surface. CD163 is one of the markers of M2 (alternatively activated) macrophages, which has been detected in diseases associated with SIRS such as Ebola virus disease (24). Hypersecretion of the immunosuppressive cytokine IL-10 from CD163 ${ }^{+}$ activated macrophages often contributes to inducing a hypoinflammatory status, referred to as compensatory antiinflammatory response syndrome, which itself triggers an abnormal, uncontrolled inflammatory dysregulation. In fact, IL-10 level is highly increased in severe/fatal SFTS $(7,8,15)$. Intriguingly, the nonstructural SFTSV protein NSs, which is known as a virulence factor, induces IL-10 production by activating the tumor progression locus 2 (TPL2) signaling pathway (25). Thus, CD163 ${ }^{+}$macrophages may play critical roles in SFTS pathogenesis, without having a major role in viral dissemination as primary target cells. On the other hand, antigen-presenting cells (DCs) are significantly involved in initiating the germinal center response to differentiate B cells, which may be a critical process for the infection of differentiated plasmablasts with SFTSV. Identification of other primary target cells at the early stage of SFTSV infection in humans that disseminate the virus and trigger systemic inflammatory responses as well as alter B cell/plasmablast susceptibility to SFTSV will be a crucial research subject to address.

In summary, the present study by Suzuki et al. made significant progress toward understanding the pathogenesis of SFTS, which is essential for developing effective countermeasures against this emerging life-threatening tick-borne hemorrhagic fever. Their remarkable work also emphasizes the importance and robustness of using a histopathology approach for studying the pathogenesis of viral disease.

Address correspondence to: Hideki Ebihara, Guggenheim 18-11A, 200 First Street SW, Rochester, Minnesota 55905, USA. Phone: 507.538.1300; Email: Ebihara. Hideki@mayo.edu.

1. Yu XJ, et al. Fever with thrombocytopenia associated with a novel bunyavirus in China. $N$ Engl $J$ Med. 2011;364(16):1523-1532.

2. Choi SJ, et al. Severe fever with thrombocytopenia syndrome in South Korea, 2013-2015. PLoS Negl Trop Dis. 2016;10(12):e0005264.

3. Takahashi $\mathrm{T}$, et al. The first identification and retrospective study of severe fever with thrombocytopenia syndrome in Japan. J Infect Dis. 2014;209(6):816-827.

4. Zhan J, et al. Current status of severe fever with thrombocytopenia syndrome in China. Virol Sin. 2017;32(1):51-62.

5. [No authors listed]. Severe fever with thrombocytopenia syndrome (SFTS) in Japan, as of June 2019. National Institute of Infectious Diseases: Tokyo, Japan. https://www.niid.go.jp/niid/en/ iasr-vol40-e/865-iasr/9021-473te.html. Accessed December 11, 2019.

6. Saijo M. Pathophysiology of severe fever with thrombocytopenia syndrome and development of specific antiviral therapy. J Infect Chemother. 2018;24(10):773-781.

7. Mendoza CA, Ebihara H, Yamaoka S. Immune modulation and immune-mediated pathogenesis of emerging tickborne banyangviruses. Vaccines (Basel). 2019;7(4):E125.

8. Sun Y, et al. Host cytokine storm is associated with disease severity of severe fever with thrombocytopenia syndrome. J Infect Dis. 2012;206(7):1085-1094.

9. Song P, et al. Deficient humoral responses and disrupted B-cell immunity are associated with fatal SFTSV infection. Nat Commun. 2018;9(1):3328.

10. Li MM, et al. CD4 T cell loss and Th2 and Th17 bias are associated with the severity of severe fever with thrombocytopenia syndrome (SFTS).
Clin Immunol. 2018;195:8-17.

11. Suzuki T, et al. Banyangvirus targets differentiating B cells in human lethal severe fever with thrombocytopenia syndrome. JClin Invest. 2020;130(2):799-812.

12. Martines RB, Ng DL, Greer PW, Rollin PE, Zaki SR. Tissue and cellular tropism, pathology and pathogenesis of Ebola and Marburg viruses. J Pathol. 2015;235(2):153-174.

13. Burt FJ, et al. Immunohistochemical and in situ localization of Crimean-Congo hemorrhagic fever (CCHF) virus in human tissues and implications for CCHF pathogenesis. Arch Pathol Lab Med. 1997;121(8):839-846.

14. Takahashi T, et al. Transient appearance of plasmablasts in the peripheral blood of Japanese patients with severe fever with thrombocytopenia syndrome. J Infect Dis. 2019;220(1):23-27.

15. Hu LF, et al. The regulation of seventeen inflammatory mediators are associated with patient outcomes in severe fever with thrombocytopenia syndrome. Sci Rep. 2018;8(1):159.

16. McElroy AK, et al. Human Ebola virus infection results in substantial immune activation. Proc Natl Acad Sci USA. 2015;112(15):4719-4724.

17. García M, et al. Massive plasmablast response elicited in the acute phase of hantavirus pulmonary syndrome. Immunology. 2017;151(1):122-135.

18. Zhang J, Yan X, Li Y, Gao R, Wang P, Mo W. Reactive plasmacytosis mimicking multiple myeloma associated with SFTS virus infection: a report of two cases and literature review. BMC Infect Dis. 2018;18(1):528.

19. Wada T, Iwata Y, Kamikawa Y, Wada T, Yachie A. Peripheral blood plasmacytosis in severe fever with thrombocytopenia syndrome. Jpn J Infect Dis. 2017;70(4):470-471.

20. Hofmann H, et al. Severe fever with thrombocytopenia virus glycoproteins are targeted by neutralizing antibodies and can use DC-SIGN as a receptor for $\mathrm{pH}$-dependent entry into human and animal cell lines. J Virol. 2013;87(8):4384-4394.

21. Geisbert TW, Jahrling PB. Exotic emerging viral diseases: progress and challenges. Nat Med. 2004;10(12 Suppl):S110-S121.

22. Jung IY, et al. Higher fatality for severe fever with thrombocytopenia syndrome complicated by hemophagocytic lymphohistiocytosis. Yonsei Med J. 2019;60(6):592-596.

23. Nakano A, et al. Hemophagocytic lymphohistiocytosis in a fatal case of severe fever with thrombocytopenia syndrome. Intern Med. 2017;56(12):1597-1602.

24. McElroy AK, et al. Macrophage activation marker soluble CD163 associated with fatal and severe Ebola virus disease in humans ${ }^{1}$. Emerging Infect Dis. 2019;25(2):290-298.

25. Choi Y, et al. Severe fever with thrombocytopenia syndrome phlebovirus non-structural protein activates TPL2 signalling pathway for viral immunopathogenesis. Nat Microbiol. 2019;4(3):429-437. 